\title{
On the Modelling of Price Effects in the Diffusion of Optional Contingent Products*
}

\author{
Guiomar Martín-Herrán ${ }^{a} \quad$ Sihem Taboubi ${ }^{b}$ \\ ${ }^{a}$ IMUVA, Universidad de Valladolid, Spain, and GERAD, Montreal, Canada. \\ ${ }^{b}$ GERAD and Marketing Department, HEC Montreal, Canada.
}

\begin{abstract}
In this chapter, we study the pricing strategies of firms in a multi-product diffusion model where we use a new formalization of the price effects. More particularly, we introduce the impact of prices on one of the factors that affect the diffusion of new products: the innovation coefficient. By doing so, we relax one of the hypotheses in the existing literature stating that this rate is constant. In order to assess the impact of this functional form on the pricing policies of firms selling optional contingent products, we use our model to study two scenarios already investigated in the multiplicative form model suggested by Mahajan and Muller (1991) (M\&M).

We follow a 'logical experimentation' perspective by computing and comparing the results of three models: (i) The M\&M model, (ii) a modified version of $M \& M$ where the planning horizon is infinite, and (iii) our model, where the new formalization of the innovation effect is introduced. This perspective allows us to attribute the differences in results to either the length of the planning horizon, or to our model's formalization. Besides its contribution to the literature on pricing and diffusion, this paper highlights the sensitivity of results to the hypothesis used in product diffusion modelling and could explain the mixed results obtained in the empirical validations of diffusion models (Mesak, 1996).
\end{abstract}

Keywords: Marketing modelling, Diffusion models, Dynamic Pricing, Contingent products.

\footnotetext{
${ }^{*}$ We are grateful to two anonymous reviewers for valuable comments and suggestions on an earlier draft of this paper. This research is partially supported by Spanish MINECO under projects ECO2014-52343-P and ECO2017-82227-P (AEI) and by Junta de Castilla y León under projects VA105G18 and VA024P17 co-financed by FEDER funds (EU).
} 


\section{Introduction}

This chapter contributes to the marketing modelling literature on products' diffusion and pricing. More particularly, we extend the existing literature that incorporated the price effects in the Bass diffusion model (Bass, 1969) by providing a new functional form that captures the negative impact of prices on the innovation rate, a key parameter in the diffusion process. By using this formalization, we investigate the pricing strategies of firms in the case where contingent products are sold in the market. Contingent products are products positively inter-related in their demands. That is, an increase in the demand of one of them contributes the demand increase of the other one. The marketing literature distinguishes between two types of product contingency: The optional contingent products and the captive contingent ones (Peterson and Mahajan, 1978; Kotler, 1988, p. 516-517). The latter case captures situations where none of the products can be used without the other, while the former case describes situations where one of the products (i.e., the contingent) is useless without the other, often denoted by "the base" (i.e., or primary) product.

In all cases, the interdependencies in products' demands highlight the importance of studying the influence of each product's price on its diffusion and the diffusion of the other product. Indeed, if a firm decides to fix a high (low) price at the launch of a new primary product in order to skim (penetrate) the market, we expect this price to have an impact on the adoption of the primary and the contingent products. These effects depend necessarily on the sensitivity of consumers to prices, which could differ depending on whether they belong to the innovators or the imitators' segments, as classified in the Bass model. Another important question to be addressed when examining the pricing strategies for interdependent products is related to the firms controlling the prices of these products: How pricing strategies compare whenever both products are sold via a single firm versus separate firms?. It has been often observed in practice and reported in the marketing literature (Kotler, 1988) that a single firm controlling the prices of two products with optional-contingent interdependencies, uses one of the products as a springboard to increase the adoption of the second one (Kort, Taboubi and Zaccour, 2018). It is not clear in this case how independent firms controlling each one of these products separately will fix their pricing strategies for the product under their control.

The pricing of contingent products by a single versus two independent firms is a topic that has been previously investigated in the study of Mahajan and Muller (1991) (M\&M). The authors used an extension of the Bass model where prices affect the base and the contingent product's demands while the diffusion parameters (i.e., innovation and imitation) are considered constant. Hence, their model does not capture the fact that prices could influence endogenously one or both of these diffusion parameters, as suggested in the literature on products diffusion and captured by the functional form that we propose in this chapter.

To the best of our knowledge, our study is one of the first to examine the pricing strategies of firms in the complex case where the innovation parameter is price-dependent, and imitation is taken into account. Indeed, the existing literature that introduced endogenously 
the price effect on the diffusion parameters considered that diffusion is driven only by innovation. The imitation effect, which implies the modelling of the interaction between the state variable representing the cumulative number of adopters of the product, the number of non-adopters ${ }^{1}$ and the control variable (i.e., price) was systematically omitted. With this simplifying assumption, the diffusion process is represented by a dynamic function which is multiplicatively separable in the state and the price, a feature that makes the model analytically tractable. The new formulation that we suggest in this study provides solutions for the more complex case where both innovation and imitation drive diffusion, and innovation is a price-dependent parameter.

Besides extending the diffusion literature to capture additional features in the diffusion process, our objectives are to assess the impact of using this new functional form and modifying the length of the planning horizon on the previous results obtained in the literature regarding the pricing policies of firms selling contingent products. To achieve these objectives, we compute prices, cumulative sales and profits for the base and the contingent products under the two scenarios investigated in M\&M: A scenario where both products are sold by the same monopolistic firm, and a scenario where two distinct firms sell each one of the products. Our results are then compared to those obtained in a modified version of the $M \& M$ model $^{2}$ where we consider an infinite planning horizon. This modified version of the M\&M model is used as a benchmark to assess the impact on results of two modelling hypotheses in the diffusion literature: The formalization of the parameters' effect and the length of the planning horizon.

The chapter is organized as follows. Section 2 revisits the Bass model, while Section 3 presents the M\&M model and its original results under a finite-time horizon. Section 4 presents our model, and Section 5 extends the results obtained by M\&M to an infinite-time horizon and compares the results obtained with this modified version to those obtained under our formulation of the price effects. Section 6 concludes.

\section{The Bass model}

The seminal paper of Bass (1969) is the first study that introduced an analytical model of diffusion in the marketing literature. According to this model, the diffusion of a new product in the market can be modelled as a dynamic process describing the evolution in the number of adopters. According to the Bass model, buyers can be either innovators or imitators. The innovators are the portion of the market potential that adopt the new product after being

\footnotetext{
${ }^{1}$ The number of non-adopters depends also on the state variable. It captures the remaining potential market at a particular time period. It is computed as the difference, in each time period, between the total market potential and the cumulative number of adopters at that time period.

${ }^{2}$ In the M\&M model, the authors study additional scenarios involving the two cases of contingency described above (i.e., optional and captive). In order to focus on the main objective of our study, we restrict our analysis to investigate only the case of optional contingent products.
} 
exposed to external factors. The remaining portion of the market is composed of imitators who adopt the new product because they are sensitive to the word-of-mouth conveyed by those who already adopted the innovation. Denoting by $x(t)$ the cumulative number of adopters by time $t \in\left[t_{0}, T\right]$, the diffusion process in Bass (1969) is captured by the following differential equation

$$
\dot{x}(t)=\left(a+\frac{b}{M} x(t)\right)(M-x(t))
$$

where $\dot{x}(t)$ denotes the adoption rate of the new product at time $t$, and $M$ is a positive parameter representing the market potential of the new product. ${ }^{3}$ In the normalized Bass model, this equation can be rewritten as

$$
\frac{\dot{x}(t)}{1-x(t)}=a+b x(t)
$$

which indicates that $(a+b x(t))$ is a hazard function representing the likelihood of adoption of a new product at time $t$ given that it has not yet been adopted. This function, called also the conversion function, depends on the positive parameters $a$ and $b$, which correspond to the innovation and the imitation rates, as well as the the cumulative number of adopters. In the early version of the Bass model, the parameters $a$ and $b$, and the market potential $M$, are considered constant, and the model is solved by considering a finite time-horizon.

By solving the differential equation given in the Bass model, it is possible not only to estimate the number of adopters at each time $t$, but also to compute the timing and the magnitude of the peak sales (or adoptions).

Despite its performance to forecast the number of adopters for various durable products, the Bass model has been criticized by many researchers in marketing management because of some of its underlying hypotheses. Indeed, various authors raised some concerns related to the use of constant parameters capturing the innovation and the imitation rates, on top of considering that the market potential of the new product does not change. Furthermore, the original version of the Bass model did not account for the impacts of the marketing efforts deployed by the firm launching the new product on its diffusion, nor the impacts of their competitors' strategies or the other products available in the market. ${ }^{4}$

Hence, over the years, this model provided a basic framework for many extensions in the product diffusion modelling literature. The main objective behind these developments was to enhance the model forecasting performance and to transform it from a time-series model into an optimization tool for decision-making.

\footnotetext{
${ }^{3}$ The state dynamics of the cumulative number of adopters when the innovation rate parameter $a$ equals zero corresponds to the well-known logistic equation usually described by $\dot{P}=r P(1-P / k)$, where $P$ is the population size, $r$ a constant that defines the growth rate and $k$ is the carrying capacity.

${ }^{4}$ For a detailed analysis of these limits, see Mahajan et al. (1990).
} 
Introducing the effect of different decision variables, such as prices and advertising is one of the developments investigated in the marketing science literature on product diffusion. Considering the interactions between multiple products is another important one. The multiple-products' diffusion models encompass cases where $(i)$ products are sold by competing firms in the market; $(i i)$ one of the products is a new generation of another one; and (iii) products are complementary or contingent. The extension of diffusion models to handle these various cases is justified by the competitive environment of firms and the fact that most of them often face situations where they have to manage various products interrelated in their demands (i.e., a product mix).

As mentioned in the introduction, our paper is an extension of the Bass (1969) model that takes into account the two development axes described above, in addition of exploring the impact of extending the length of the planning horizon. We investigate the product diffusion process by introducing prices as decision variables in an innovative manner, and by considering the interactions between two products. More particularly, we investigate the case of optional contingent products. This paper goes in the same vein than the M\&M study who examined the same issue with a different model's formalization of the price effects on diffusion. In the M\&M model, prices affect the diffusion dynamics in a multiplicative separable manner, as suggested initially by Robinson and Lakhani (1975) and generalized through the $\mathrm{GBM}^{5}$ by Bass et al. (1994). This formulation captures the fact that prices have an effect on the probability of purchase of non-adopters, rather than on the model's key parameters, that is: The market potential and the innovation and imitation rates. Hence, prices can shift the probability of purchase upward or downward, and consequently, speed or slow down the adoption, but do not influence in an endogenous manner the number of innovators or imitators, nor the total market potential.

Because the multiplicative model formalization introduces the price effect in the diffusion process without affecting the model's parameters, the GBM model remains analytically tractable, even when multiple firms selling competing or contingent products are considered in the market. This mathematical convenience allowed this functional form to be widely used in the diffusion literature (Jørgensen and Zaccour, 2004, p. 73). The general result obtained with this formalization states that, when the imitation effect is high, a firm should use a low price for the launch of its new product in order to attract innovators. The price can be increased later when the product is established and then decreased again.

When the price effects in the diffusion model are introduced by varying the market

\footnotetext{
${ }^{5}$ i.e., GMB states for the General Bass Model. The GBM is an extension of the Bass model that incorporates, in a multiplicative way, the effect of the marketing variables. According to this model, diffusion is described by the following differential equation:

$$
\dot{x}(t)=(a+b x(t))(M-x(t)) g(V),
$$

with $g(V)$ representing a function capturing the impact of firms' decision variables (eg. advertising, price, etc.)
} 
potential, a skimming strategy where prices are fixed at a high level at the introductory phase is optimal when the imitation effect is low, while a penetration strategy is recommended when the imitation effect is high (Horsky, 1990).

As mentioned in the introductory section, our formalization of the price impact on diffusion is introduced in our model through the innovation rate. We model this parameter as a price-dependent function which drives an increase in the innovation rate whenever a firm decides to lower its price (i.e., a penetration strategy) or a decrease in the opposite case (i.e., a skimming strategy).

The choice of this formalization can be justified from a managerial point of view: Firms often target innovators when they choose their marketing strategies since innovators are the early adopters of the new product, and they have the power of influencing the buying decisions of imitators (Jørgensen and Zaccour, 2004, p. 49). The study of Mesak (1996) provides an empirical validation of this hypothesis with real data. Indeed, the author tested various diffusion models incorporating price, advertising and distribution in order to identify which one fits better the data on the diffusion of cable TV. One of its main findings is that, for this innovation, the model that performs better is the one where price affects only the innovation rate, advertising affects the diffusion rate, ${ }^{6}$ and distribution affects the market potential.

Most of the studies that considered the case where the diffusion model's parameters are endogenous focused on firms' advertising strategies while only few of them used a similar formulation for pricing decisions. The advertising effect, considered as positive, has been introduced either through the innovation rate only (Horsky and Simon, 1983) or through both the innovation and the imitation rates (Dockner and Jørgensen, 1988; Teng and Thompson, 1983). In Thompson and Teng (1984), the authors introduced both the price and the advertising effects in the diffusion process. They considered that advertising affects both the innovation and the imitation rates, while prices have a multiplicative effect that shifts all the diffusion function as in the BGM. Additional extensions of the Bass model incorporated also the impact of economic conditions and marketing decision variables (eg., distribution) on the market potential (Mahajan et al., 1979; Jones and Ritz, 1991, Kalish, 1985). ${ }^{7}$

\section{The Mahajan and Muller (1991) Model}

The first adaptation of the Bass model to the multi-product context, and more particularly to the case of contingent products' demands is presented in Peterson and Mahajan (1978). Mahajan and Muller (1991) provided an additional extension of this model by introducing the price effects according to a multiplicative-form model, as in the GBM.

\footnotetext{
${ }^{6}$ The effect here is found to be multiplicative, as in the GBM, meaning that advertising affects equally the innovation and the imitation rates.

${ }^{7}$ See Peres et al. (2010) for a more recent review of diffusion models.
} 
The authors considered a market where two products $i=\{1,2\}$ are sold at prices $p_{i}(t)$ : Product 1 is a base (or primary) product that could be bought independently by consumers, while product 2 is a contingent product purchased only by consumers who al ready bought the base product. By letting $x_{1}(t)$ and $x_{2}(t)$ denote the cumulative sales of the base and the contingent products respectively, and $M$ represent the market potential of these products. The instantaneous sales for both products are described by the following dynamic equations:

$$
\begin{aligned}
& \dot{x}_{1}(t)=\left(a_{1}+b_{1} x_{1}(t)\right)\left(M-x_{1}(t)\right) e^{-\epsilon_{1} p_{1}(t)}, \\
& \dot{x}_{2}(t)=\left(a_{2}+b_{2} x_{2}(t)\right)\left(x_{1}(t)-x_{2}(t)\right) e^{-\epsilon_{2} p_{2}(t)},
\end{aligned}
$$

where $a_{i}$ and $b_{i}$ represent the product $i$ 's innovation and imitation parameters, respectively, and $\epsilon_{i}$ denote the price sensitivity parameter of product $i$.

The first differential equation is the standard diffusion equation used in the GBM, where $g(V)$ is a price-dependent function. It describes the adoption process of the base product (i.e., product 1 ) through the effects of innovation $\left(a_{1}\right)$, imitation $\left(b_{1}\right)$, and retail price. The main feature of equation (1) is that it is independent from equation (2), meaning that the diffusion of the base product is not affected by the diffusion of the contingent product. This characteristic captures the one-way demand interdependencies between both products in the case of an optional contingency relationship.

The second equation describes a similar process for the contingent product (i.e., product 2 ), but features a dependence of the contingent-product's diffusion on the diffusion of the base product. We can clearly see that the market potential for the contingent product is determined by the current cumulative sales volume of the base product. As in equation (1), equation (2) indicates that, at each time $t$, the number of potential buyers of the contingent product corresponds to the total number of buyers of the base product who did not previously bought the contingent product. ${ }^{8}$ Here again, the adoption of the contingent product is subject to the impact of the constant parameters of innovation and imitation, and the product's retail price.

By considering a finite time horizon and a production cost $\left(c_{i}\right)$, the authors computed and compared price trajectories, sales, and profits for both products under the scenarios where they are controlled by the same ${ }^{9}$ or by different firms in order to test the following propositions: ${ }^{10}$

- Proposition A: When both products are sold by the same firm (i.e., an integrated monopolist), the firm sets a lower price on the base product and a higher price on the

\footnotetext{
${ }^{8}$ Hence, the maximum number of consumers who could buy the contingent product should not exceed the maximum number of consumers who already bought the base product. (i.e. the market potential $M$ ).

${ }^{9}$ We use the superscript $m$ to denote the case of an integrated monopolist (i.e., a single firm selling both products).

${ }^{10}$ Remark: In the case of captive contingency, the price effect is introduced by considering that each one of the product diffusion processes is affected by not only its own price, but also by the price of the other product.
} 
contingent one, with respect to the case where both products are sold by independent firms. This translates into the following inequalities

$$
p_{1}^{m}(t)<p_{1}(t) \quad \text { and } \quad p_{2}^{m}(t)>p_{2}(t) \text {. }
$$

- Proposition B: When both products are sold by the same firm, most of the profit is earned from the contingent product rather than the base one, and the markup for this product is set at a higher level. Hence, by using the notation $z_{i}$ to designate the total profit generated by product $i$ in the integrated monopolist scenario, this proposition translates into the following inequalities

$$
z_{1} /\left(z_{1}+z_{2}\right)<z_{2} /\left(z_{1}+z_{2}\right) \quad \text { and } \quad\left(p_{1}-c_{1}\right)<\left(p_{2}-c_{2}\right) .
$$

To test these propositions, the authors solved both scenarios by using in the first case an optimal control problem, where the unique decision maker (i.e., the monopolistic firm) chooses the two control variables $\left(p_{1}, p_{2}\right)$. His objective is to maximize his total discounted profit while taking into account the dynamics of the two state variables $x_{1}, x_{2}$. The optimality conditions are a system of four differential equations with two initial conditions for the state variables $x_{1}$ and $x_{2}$ and two final conditions for the corresponding costate variables.

In the case of the independent producers, on the one hand, the primary producer, solves a control problem, where he decides its control variable $p_{1}$ with the objective of maximizing his discounted cumulative profits while taking into account the state variable $x_{1}$. The optimality conditions are a system of two differential equations with one initial condition for variable $x_{1}$ and one final condition for the corresponding costate variable. On the other hand, the contingent-product producer solves a control problem where he decides his control variable $p_{2}$ in order to maximize his objective functional taking into account the state variables $x_{1}$ and $x_{2}$. The optimality conditions in this case are a system of three differential equations with two initial conditions for variables $x_{1}$ and $x_{2}$ and one final condition for one costate variable. Putting together the optimality conditions for both firms (primary and contingent products' producers), these conditions constitute a system of four differential equations with two initial conditions for variables $x_{1}$ and $x_{2}$ and two final conditions for the corresponding costate variables. Both problems are solved by considering a finite-horizon and the resolution is made numerically.

The numerical simulations are made by considering the following parameters' values:

$$
\begin{aligned}
& a_{1}=a_{2}=0.015, \quad b_{1}=b_{2}=.4 / 60.000, \quad c_{1}=c_{2}=60, \quad r=0.01, \\
& \varepsilon_{1}=\varepsilon_{2}=0.01, \quad M=60.000, \quad x_{10}=6.000, \quad x_{20}=0 .
\end{aligned}
$$

The authors analyzed the steady state of the different scenarios as well as the optimal paths converging to the steady state. In the two scenarios, both $x_{1}$ and $x_{2}$ take the value 
$M$ at the steady state. That is, along all the optimal paths both $x_{1}(t)$ and $x_{2}(t)$ converge towards $M$ (See note 8).

The results obtained by M\&M provided only a partial validation of proposition A and failed to support proposition B. Indeed, the authors were not able to prove, for any instant of time $t$, that the integrated monopolist producer will fix a lower price for the primary product and a higher price for the contingent one, while the independent producers will do the opposite (i.e., proposition A). The analytical results proved that $p_{1}^{m}<p_{1}$ for the same level of penetration ( i.e., $x_{1}=x_{1}^{m}$ ) only when the time horizon $T$ is large. Furthermore, they found an opposite result with respect to the pricing of the contingent product. Indeed, their results indicate that the integrated monopolist fixes a lower price for the contingent product, when compared to the price that an independent firm would fix for that product. This result is obtained for the same level of penetration, when $T$ is large, and the discount rate $(r)$ is small.

Nevertheless, the authors proved that the diffusion of both the base and the contingent products are faster when both products are controlled by the same firm.

Proposition B was tested by running numerous simulations. The results obtained did not support anyone of the hypotheses in this proposition. Indeed, the authors found that, in many solutions (for certain time periods), the markup for the primary product was higher than for the contingent product, but it was also lower in other cases. ${ }^{11}$ Furthermore, the base product is found to contribute more to total profits with respect to the contingent one.

\section{Our Model}

In our model, we examine the same issue on the pricing of contingent products and investigate the same scenarios as in the M\&M's model. The main difference is that in M\&M, the innovation rate is a constant parameter, while in our model, we specify a functional form that allows the innovation rate to vary through the impact of price decisions. Indeed, the functional form that we use takes explicitly into account the negative effect of product's price on early-adopters of the new product (i.e., innovators). The innovation rate is then given by the following equation

$$
a_{i}\left(p_{i}(t)\right)=\alpha_{i}-\beta_{i} p_{i}(t)
$$

where $\alpha_{i}$ and $\beta_{i}$ are positive parameters. Parameter $\alpha_{i}$ can be interpreted as a price-independent innovation rate (as in the original Bass model) that captures the fact that a portion of innovators will adopt the new product regardless of its price. Parameter $\beta_{i}$ reflects price-sensitivity of innovators. Notice that when $\beta_{1}=0$, the original Bass model is obtained, which ignores

\footnotetext{
${ }^{11}$ This result indicates that $p_{1}^{m}$ can be greater or lower than $p_{2}^{m}$ for some time periods because, as mentioned above, M\&M consider the symmetric scenario with respect to the parameters, including the symmetry in production costs (i.e., $c_{1}=c_{2}$ ).
} 
this price effect on innovators.

The dynamics of the cumulative adoption of the primary product, $x_{1}$, and the contingent product, $x_{2}$, can be written as follows: ${ }^{12}$

$$
\begin{aligned}
& \dot{x}_{1}(t)=\left(\alpha_{1}-\beta_{1} p_{1}(t)+b_{1} x_{1}(t)\right)\left(M-x_{1}(t)\right), \quad x_{1}(0)=x_{10}, \\
& \dot{x}_{2}(t)=\left(\alpha_{2}-\beta_{2} p_{2}(t)+b_{2} x_{2}(t)\right)\left(x_{1}(t)-x_{2}(t)\right), \quad x_{2}(0)=x_{20} .
\end{aligned}
$$

Because the model is not analytically tractable when a finite-time horizon is considered, we studied the problem with infinite horizon. Therefore, the objective in the case of the integrated monopolist is to choose the prices, $p_{1}$ and $p_{2}$, in order to maximize the following functional:

$$
\int_{0}^{\infty} e^{-r t}\left[\left(p_{1}(t)-c_{1}\right) \dot{x}_{1}(t)+\left(p_{2}(t)-c_{2}\right) \dot{x}_{2}(t)\right] d t
$$

taking into account (6) and (7).

In the second scenario where two independent producers control the pricing decisions of the primary and the contingent product, we have two objective functionals: the objective of the primary-product producer and the objective of the contingent-product producer.

The objective for the primary-product's producer is to choose the price $p_{1}$ in order to maximize the following functional:

$$
\int_{0}^{\infty} e^{-r t}\left(p_{1}(t)-c_{1}\right) \dot{x}_{1}(t) d t
$$

taking into account (6).

The contingent-product's producer chooses the price $p_{2}$ in order to maximize the following objective functional

$$
\int_{0}^{\infty} e^{-r t}\left(p_{2}(t)-c_{2}\right) \dot{x}_{2}(t) d t
$$

taking into account (6) and (7).

Hence, the problems that we examine are solved according to the same procedure as the one used in the M\&M model and described in the previous section. In appendix $\mathbf{A}$, we provide a detailed description of the different steps used in the resolution of both scenarios.

As mentioned in the introduction, our study allows us to attain two objectives: The primary objective is to assess the impact of using a new functional form capturing the price effects on diffusion. A second objective is to investigate the sensitivity of results to the length of the planning horizon. In order to remove any confusion between the original M\&M model solved under a finite horizon and its counterpart when the planning horizon is infinite, we designate by "Modified M\&M model" all the results

\footnotetext{
${ }^{12}$ Initially $\mathrm{M} \& \mathrm{M}$ assume $x_{20}=0$, but in their numerical simulations it seems that they consider other initial values for variable $x_{2}$ positive but lower than the initial value for the variable $x_{1}$.
} 
obtained when the M\&M model is solved under an infinite planning horizon. This modified version of the M\&M model allowed us to assess the impact of the length of the planning horizon. Indeed, by comparing the results obtained in the original study of M\&M where the time horizon is finite with those obtained with the modified M\&M model, and by using the same parameters' values, we fulfill the secondary objective. The latter is an important step to fulfill the primary objective. Indeed, by comparing the results of our model, where the planning horizon is infinite, with the modified version of the M\&M model, and by using the same parameters' values, we removed any explanation of the difference in results between the M\&M results and the results obtained with our model, which is not related to the model's functional form.

\section{Results}

In this section, we present the results obtained with the three models described above, that is, the original M\&M model, its modified version, and our suggested model, where a price-dependent innovation parameter is introduced in the diffusion process. We compare and contrast these results in order to assess the impact of using a different planning horizon and a new model's formalizations of the priceeffects. In all cases, we start by reporting in a bullet-point form the "technical" results about the number of equilibria and the analytical tractability of the models; then present the main managerial findings related to the comparisons of pricing strategies, products' diffusion, and firms' profits under the two investigated. All the results are compared to the results obtained under the original version of the M\&M model.

\subsection{Results under the modified M\&M Model}

When the M\&M model is solved under an infinite-time horizon, we find that, for both scenarios investigated:

- The dynamical system of the optimality condition only presents a unique steady state, and there is a unique optimal path converging to this steady state.

- There are two possible cases depending on whether the Jacobian matrix of the dynamical system evaluated at the steady state presents two different negative eigenvalues or presents only one double negative eigenvalue. However, when we follow the symmetry hypothesis in M\&M to analyze the particular case where all parameters are identical for both products, the Jacobian matrix of the dynamical system evaluated at the steady state in this particular case presents only one double negative eigenvalue.

- The steady-state values of the two scenarios can be analytically compared. The result 
of the comparison at the steady state (denoted by the subscript $s s$ ) is as follows:

$$
x_{1 s s}^{m}=x_{1 s s} ; \quad x_{2 s s}^{m}=x_{2 s s} ; \quad p_{1 s s}^{m}<p_{1 s s} ; \quad p_{2 s s}^{m}=p_{2 s s} .
$$

As in the original M\&M model, we test propositions $\mathrm{A}$ and $\mathrm{B}$ by running numerical simulations where we use the parameters' values given in (3) and (4). The state and price trajectories under both scenarios of the modified M\&M model can be illustrated in Figure 1 and Figure 2. ${ }^{13}$

\section{-INSERT FIGURE 1 HERE-}

Figure 1 indicates that, under both scenarios, the optimal cumulative adoption paths for both products (i.e., $x_{1}(t)$ and $x_{2}(t)$ ) increase monotonously towards the market potential level $M$ with $x_{1}(t)$ always higher than $x_{2}(t)$ for all $t$. As already obtained in M\&M in a finite horizon, our results indicate that, under an infinite time horizon, the diffusion of the base and the contingent products are faster when they are sold by the same firm.

\section{-INSERT FIGURE 2 HERE-}

Figure 2 shows that under the two scenarios, both the optimal price paths $p_{1}(t)$ and $p_{2}(t)$ increase monotonously towards their steady state values. This result differs from its counterpart in the original M\&M model since the authors found that the price of the primary product could drop when a finite planning horizon is considered.

Furthermore, we can see from this figure that in the integrated monopolist case, $p_{2}^{m}(t)>$ $p_{1}^{m}(t)$ for all $t$, while in the independent producers scenario $p_{1}(t)>p_{2}(t)$ for all $t$. Figure 2 shows also that $p_{1}(t)>p_{1}^{m}(t)$ for all $t$, and $p_{2}(t)<p_{2}^{m}(t)$ for all $t$, except for a very short initial period of time. This indicates that the result on the price comparisons for the contingent product provided in Proposition A is always supported when an infinite time horizon is considered.

With the numerical results obtained under the modified M\&M model, we computed the markups and the relative profits of the base and the contingent products under the two scenarios investigated. Our results were qualitatively similar to those obtained under a finite-time horizon and did not support proposition B.

Hence, when compared to the original M\&M model, the results obtained under the modified M\&M model allow us to conclude that moving from a finite to

\footnotetext{
${ }^{13}$ Similar figures have been computed for the following cases:

- $x_{20}=3.000,6.000$;

- $M=40.000,70.000,80.000$;

- $\varepsilon_{1}=\varepsilon_{2}=0.02$.
}

showed qualitatively similar results. 
an infinite planning horizon has an impact on firms strategies. Indeed, although the results on adoption levels and profit markups seem to coincide under both settings, the optimal pricing strategies of firms differ.

\subsection{Results with our model}

From a technical point of view (see Appendix A for more details), our model indicates that:

- For both scenarios, and by using the same configuration of the parameters, the dynamical system of the optimality condition presents four different steady states. Depending on the steady state, it can be either $(i)$ a unique optimal path converging to the steady state; (ii) a one-parametric family of solutions converging to the steady state; or (iii) a bi-parametric family of solutions converging to the steady state.

From the four possible steady states, we disregard three of them because one of the following reasons:

- For one of the steady states, the family of solutions converging to this steady state imposes that $x_{1}(t)=M, x_{2}(t)=M$ for all $t$ (which seems to be an uninteresting case).

- A second steady state is removed from the analysis because, along the optimal paths converging to this steady state, it imposes that $x_{1}(t)=M$ for all $t$.

- A third steady state is disregarded because a relationship among the initial values of the state variables, $x_{10}$ and $x_{20}$ is needed to ensure the convergence of the optimal paths to the steady state. This relationship can be viewed as a "knife-edge condition".

Therefore, we focus only on the fourth steady state for which there exists a unique optimal path converging to this steady state.

The managerial results indicate that, at this steady-state, the price of the contingent product under the integrated monopolist is identical to its price under the independent firms' scenario, while prices at the steady-state differ among the scenarios when we turn to the primary product:

$$
x_{1 s s}^{m}=x_{1 s s} ; \quad x_{2 s s}^{m}=x_{2 s s} ; p_{1 s s}^{m}<p_{1 s s} ; p_{2 s s}^{m}=p_{2 s s} .
$$

That is, we have that in the long run (i.e. at the steady state) the comparison of the two scenarios (integrated monopolist and independent producers) is qualitatively similar with respect to the modified $\mathrm{M} \& \mathrm{M}$ model ${ }^{14}$.

\footnotetext{
${ }^{14}$ However, the transitional dynamics, that is, the transition towards these steady states could be different.
} 
As mentioned above, our numerical simulations are based on the same parameters' values as in the M\&M's paper. Furthermore, we have fixed the following values of the parameters which are specific of our model's functional form:

$$
\alpha_{1}=\alpha_{2}=30, \beta_{1}=\beta_{2}=0.1
$$

For these values of the parameters and in all the numerical simulations we have carried out we have checked that the innovation rate described in equation (5) is between zero and one for both products.

Figure 3 and Figure 4 have been generated by solving our model and using the values of the parameters in (3) and (4) when applicable together with the values of parameters $\alpha$ 's and $\beta$ 's above. ${ }^{15}$

\section{-INSERT FIGURE 3 HERE-}

From Figure 3 we have that under the two scenarios, the optimal cumulative adoption paths for both products increase monotonously towards the market potential level for all $t$, and $x_{1}(t)$ is always higher than $x_{2}(t)$.

\section{-INSERT FIGURE 4 HERE-}

Figure 4 indicates that under the two scenarios, both the optimal price paths $p_{1}(t)$ and $p_{2}(t)$ increase monotonously towards their steady-state values. In the independent producers scenario $p_{2}(t)<p_{1}(t)$ for all $t$; while in the integrated monopolist case, $p_{1}^{m}(t)<p_{2}^{m}(t)$ for all $t$. Furthermore, Figure 4 shows for our model that $p_{1}(t)>p_{1}^{m}(t)$ and $p_{2}(t)<p_{2}^{m}(t)$ for all $t$. Therefore, the result of Proposition $\mathrm{A}$ in M\&M holds for the price of both the contingent and primary products.

Concerning the first hypothesis in Proposition B, which was rejected in both the M\&M and the modified M\&M models, with our new formalization of the price effect, we found that the same result as in these models is obtained (i.e., $\left.z_{1} /\left(z_{1}+z_{2}\right)>z_{2} /\left(z_{1}+z_{2}\right)\right)$. Indeed, we found that by keeping fixed the parameters in (3), (4) and (8) and by changing the initial values of the cumulative adoption of the contingent product $\left(x_{20} \in\{0,3.000,6.000\}\right)$, Proposition B is always rejected. Furthermore, we run some additional simulations by changing the value of the market potential $M$ and found that this value does not have an impact on this result. Independently of the value of the market potential $M(M=40.000, M=$ 80.000) Proposition B is rejected.

\footnotetext{
${ }^{15}$ Qualitatively similar figures have been obtained for the following cases:

- $x_{20}=3.000,6.000$;

- $M=40.000,80.000$.
} 
Hence, these results are qualitatively similar to those obtained in the modified M\&M model, which indicates that the introduction of a price-dependent innovation parameter in the diffusion model does not have a qualitative impact on the previous results obtained with diffusion models where the innovation effect is considered as a constant parameter.

\section{Conclusion}

In a paper on theoretical modeling in marketing, Moorthy (1993) provides a "logical experimentation" perspective on the building of mathematical theories in this field. This perspective is borrowed from the behavioral marketing research where empirical experiments are used to test the cause-effects relationships that could be observed between a phenomena under study, and other variables that could explain it. This process starts by building a first theoretical model to capture and explain a phenomena (i.e., the dependent variable) under a set of hypothesis. The latter could have impacts on the results and can be seen as independent variables. By modifying these hypotheses one by one, different models are tested as various treatments in a series of experiments, and by comparing the results obtained under the different models, one can understand the cause-effect relationship between the phenomena under investigation and its hypotheses and assess their impacts.

In this paper, we contribute to the modeling literature in marketing by following the logical experimentation perspective suggested by Moorthy (1993). By examining the same phenomena describing the diffusion of optional-contingent products with the M\&M model, we started by extending its results from a finite to an infinite time horizon in order to isolate the effects of the planning horizon on the results. Then, we used this modified M\&M model and compared its results to those obtained with a model where the innovation rate is modeled as a price-dependent variable. We were able to assess the impact of using this new model formalization on the multi-product diffusion phenomena and the firms strategies and profits.

Diffusion models have been widely studied since the introduction of the Bass model in the marketing science literature. Despite all the efforts to extend this model and to relax some of its limiting assumptions, there are still many open questions on the diffusion process. What marketing variables affect the adoption of new products, and how to model their effects on adoption are some of these open-questions. Mesak (1996) established this in a study where he classified most of the models that incorporate the effects of marketing variables in the Bass model and tested empirically the validity for some of them. He concluded his study by stating that "The issue of how marketing mix variables such as price, advertising and distribution affect the diffusion of innovations continues to be a debated issue in the literature" (Mesak, 1996, p. 1011). This paper can be seen as an additional treatment in the product diffusion literature where the effect of modeling differently the price impact

on diffusion and extending the planning horizon are investigated. An interesting 
extension of this study is to test the external validity of these multi-product models as in Mesak (1996) by performing their empirical validation with real market data on sales and prices of new products characterized by optional contingencies in their demands.

\section{Appendix A}

In the scenario where the independent producers control the pricing decisions of the primary and contingent products, the objective for the primary-product's producer is to choose the price $p_{1}$ in order to maximize the following functional:

$$
\int_{0}^{\infty} e^{-r t}\left(p_{1}(t)-c_{1}\right) \dot{x}_{1}(t) d t
$$

taking into account (6).

In order to find the first-order conditions necessary for optimality, we construct the current value Hamiltonian:

$$
H^{1}\left(x_{1}, p_{1}, \lambda_{1}\right)=\left(p_{1}-c_{1}\right) \dot{x}_{1}+\lambda_{1} \dot{x}_{1}=\left(p_{1}-c_{1}+\lambda_{1}\right) \dot{x}_{1},
$$

where $\lambda_{1}$ denotes the costate variable associated with $x_{1}$.

The maximization of $H^{1}$ with respect to $p_{1}$ yields $\partial H^{1} / \partial p_{1}=0$, and assuming $x_{1}$ is different from $M$, from this condition one gets:

$$
p_{1}=\frac{\alpha_{1}+\left(c_{1}-\lambda_{1}\right) \beta_{1}+b_{1} x_{1}}{2 \beta_{1}} .
$$

The Maximum Principle optimality conditions also include

$$
\begin{aligned}
& \dot{\lambda}_{1}=r \lambda_{1}-\frac{\partial H^{1}}{\partial x_{1}}, \quad \lim _{t \rightarrow \infty} \lambda_{1}(t) x_{1}(t) e^{-r t}=0, \\
& \dot{x}_{1}=\left(\alpha_{1}-\beta_{1} p_{1}+b_{1} x_{1}\right)\left(M-x_{1}\right), \quad x_{1}(0)=x_{10} .
\end{aligned}
$$

This boundary value problem taking into account expression (9) reads:

$$
\begin{aligned}
& \dot{\lambda}_{1}=\frac{1}{4 \beta_{1}}\left[\left(3 x_{1}-2 M\right) x_{1} b_{1}^{2}+\left(\alpha_{1}-\left(c_{1}-\lambda_{1}\right) \beta_{1}\right)\left(\alpha_{1}-\left(c_{1}-\lambda_{1}\right) \beta_{1}+2 b_{1}\left(2 x_{1}-M\right)\right)+4 r \beta_{1} \lambda_{1}\right] \\
& \dot{x}_{1}=\frac{1}{2}\left(M-x_{1}\right)\left(\alpha_{1}-\left(c_{1}-\lambda_{1}\right) \beta_{1}+b_{1} x_{1}\right) .
\end{aligned}
$$

Function $H^{1}$ is concave with respect to $p_{1}$.

The objective for the contingent-product's producer is to choose the price $p_{2}$ in order to 
maximize the following functional:

$$
\int_{0}^{\infty} e^{-r t}\left(p_{2}(t)-c_{2}\right) \dot{x}_{2}(t) d t
$$

taking into account the differential equations describing the dynamics of the cumulative adoption of the primary and contingent products (6) and (7) respectively.

The current value Hamiltonian reads:

$$
H^{2}\left(x_{2}, p_{2}, \lambda_{2}\right)=\left(p_{2}-c_{2}\right) \dot{x}_{2}+\lambda_{2} \dot{x}_{2}=\left(p_{2}-c_{2}+\lambda_{2}\right) \dot{x}_{2},
$$

where $\lambda_{2}$ denotes the costate variable associated with $x_{2}$.

Assuming that $x_{1}$ is different from $x_{2}$, from the optimality condition $\partial H^{2} / \partial p_{2}=0$, one gets:

$$
p_{2}=\frac{\alpha_{2}+\left(c_{2}-\lambda_{2}\right) \beta_{2}+b_{2} x_{2}}{2 \beta_{2}} .
$$

The Maximum Principle optimality conditions also include

$$
\begin{aligned}
& \dot{\lambda}_{2}=r \lambda_{2}-\frac{\partial H^{2}}{\partial x_{2}}, \quad \lim _{t \rightarrow \infty} \lambda_{2}(t) x_{2}(t) e^{-r t}=0, \\
& \dot{x}_{2}=\left(\alpha_{2}-\beta_{2} p_{2}+b_{2} x_{2}\right)\left(x_{1}-x_{2}\right), \quad x_{2}(0)=x_{20} .
\end{aligned}
$$

Substituting the expression of $p_{2}$ given by (10) into this system of differential equations we get:

$$
\begin{aligned}
& \dot{\lambda}_{2}=\frac{1}{4 \beta_{2}}\left[\left(3 x_{2}-2 x_{1}\right) x_{2} b_{2}^{2}+\left(\alpha_{2}-\left(c_{2}-\lambda_{2}\right) \beta_{2}\right)\left(\alpha_{2}-\left(c_{2}-\lambda_{2}\right) \beta_{2}+2 b_{2}\left(2 x_{2}-x_{1}\right)\right)+4 r \beta_{2} \lambda_{2}\right], \\
& \dot{x}_{2}=\frac{1}{2}\left(x_{1}-x_{2}\right)\left(\alpha_{2}-\left(c_{2}-\lambda_{2}\right) \beta_{2}+b_{2} x_{2}\right) .
\end{aligned}
$$

Function $H^{2}$ is concave with respect to $p_{2}$.

The characterization of the optimal time paths of the cumulative sales and prices of both products requires the solution of the differential equations for the state and costate variables $x_{1}, x_{2}, \lambda_{1}$ and $\lambda_{2}$. First of all, we focus on the characterization of the steady-state values and their asymptotically stability.

Because $\alpha_{i}-\beta_{i} p_{i}+b_{i} x_{i}$ for $i=2,2$ are strictly positive, the unique steady-state value of the cumulative sales is given by $x_{1 s s}=M$ and $x_{2 s s}=x_{1 s s}=M$. Taking these values into account, we compute the steady-state values of the costate variables $\lambda_{1}$ and $\lambda_{2}$. It can be easily proved that the system of differential equations admits the following four different 
steady-state values:

$$
\begin{aligned}
& \left(x_{1 s s}^{(1)}, \lambda_{1 s s}^{(1)}, x_{2 s s}^{(1)}, \lambda_{2 s s}^{(1)}\right)=\left(M, \lambda_{1}^{(1)}, M, \lambda_{2}^{(1)}\right), \\
& \left(x_{1 s s}^{(2)}, \lambda_{1 s s}^{(2)}, x_{2 s s}^{(2)}, \lambda_{2 s s}^{(2)}\right)=\left(M, \lambda_{1}^{(1)}, M, \lambda_{2}^{(2)}\right), \\
& \left(x_{1 s s}^{(3)}, \lambda_{1 s s}^{(3)}, x_{2 s s}^{(3)}, \lambda_{2 s s}^{(3)}\right)=\left(M, \lambda_{1}^{(2)}, M, \lambda_{2}^{(1)}\right), \\
& \left(x_{1 s s}^{(4)}, \lambda_{1 s s}^{(4)}, x_{2 s s}^{(4)}, \lambda_{2 s s}^{(4)}\right)=\left(M, \lambda_{1}^{(2)}, M, \lambda_{2}^{(2)}\right),
\end{aligned}
$$

where

$$
\begin{aligned}
& \lambda_{i}^{(1)}=-\frac{1}{\beta_{i}}\left(\alpha_{i}-c_{i} \beta_{i}+2 r+b_{i} M+2 \sqrt{r\left(\alpha_{i}-c_{i} \beta_{i}+r+b_{i} M\right)}\right), \\
& \lambda_{i}^{(2)}=-\frac{1}{\beta_{i}}\left(\alpha_{i}-c_{i} \beta_{i}+2 r+b_{i} M-2 \sqrt{r\left(\alpha_{i}-c_{i} \beta_{i}+r+b_{i} M\right)}\right), \quad i=1,2 .
\end{aligned}
$$

In order to analyze the stability of the steady states we compute the eigenvalues and associated eigenvectors of the Jacobian matrix evaluated at each of the steady states.

At the first steady state $\left(x_{1 s s}^{(1)}, \lambda_{1 s s}^{(1)}, x_{2 s s}^{(1)}, \lambda_{2 s s}^{(1)}\right)$ the Jacobian matrix has two negative eigenvalues and it can be proved that there exists a bi-parametric family of solutions converging to this steady state. This family of solutions imposes that $x_{1}(t)=M, x_{2}(t)=M$ for all $t$.

At the second steady state $\left(x_{1 s s}^{(2)}, \lambda_{1 s s}^{(2)}, x_{2 s s}^{(2)}, \lambda_{2 s s}^{(2)}\right)$ the Jacobian matrix has two negative eigenvalues and it can be proved that there exists a one-parametric family of solutions converging to this steady state. This family of solutions imposes that $x_{1}(t)=M$ for all $t$.

At the third steady state $\left(x_{1 s s}^{(3)}, \lambda_{1 s s}^{(3)}, x_{2 s s}^{(3)}, \lambda_{2 s s}^{(3)}\right)$ the Jacobian matrix has two negative eigenvalues and it can be proved that a relationship among the initial values of the state variables, $x_{10}$ and $x_{20}$ is needed to ensure the convergence of the optimal paths to this steady state.

At the fourth steady state $\left(x_{1 s s}^{(4)}, \lambda_{1 s s}^{(4)}, x_{2 s s}^{(4)}, \lambda_{2 s s}^{(4)}\right)$ the Jacobian matrix has two negative eigenvalues and it can be proved that there exists a unique optimal path converging to this steady state.

The numerical simulations carried out focus on this fourth scenario. In this case, the two negative eigenvalues are given by

$$
\mu_{i}=r-\sqrt{r\left(\alpha_{i}-c_{i} \beta_{i}+r+b_{i} M\right)}, i=1,2 .
$$

Following M\&M, the values of the model parameters used in the numerical simulations are assumed to be completely symmetric. Consequently, under this assumption there is a double negative eigenvalue, $\mu=\mu_{i}, i=1,2$. We have computed the associated generalized eigenvectors denoted by $\bar{v}_{1}=\left(v_{1}^{(1)}, v_{1}^{(2)}, 0,1\right)$ and $\bar{v}_{2}=\left(v_{2}^{(1)}, v_{2}^{(2)}, 1,0\right)$, with $v_{i}^{(j)}$ the $j$-th component of the $i$-th eigenvector (omitted for brevity). The solution of the system of 
differential equations read:

$$
\begin{aligned}
x_{1}(t) & =\left(x_{10}-M\right) e^{\mu t}+M, \\
\lambda_{1}(t) & =w_{1}^{(2)} e^{\mu t}+\lambda_{1 s s}^{(4)}, \\
x_{2}(t) & =\left(x_{20}-M\right) e^{\mu t}+w_{2}^{(3)} t e^{\mu t}+M, \\
\lambda_{2}(t) & =w_{1}^{(4)} e^{\mu t}+w_{2}^{(4)} t e^{\mu t}+\lambda_{1 s s}^{(4)},
\end{aligned}
$$

where $w_{i}^{(k)}$ is the $k$-th component of vector $\bar{w}_{i}$, with

$$
\bar{w}_{1}=\varphi \bar{v}_{1}+\eta \bar{v}_{2}, \quad \bar{w}_{2}=\left(\Omega-\mu I_{4}\right) \bar{w}_{1},
$$

and

$$
\varphi=\frac{1}{v_{1}^{(1)}}\left(x_{10}-M-\left(x_{20}-M\right) v_{2}^{(1)}\right), \quad \eta=x_{20}-M .
$$

Matrices $\Omega$ and $I_{4}$ denote the Jacobian matrix associated to the system of differential equations evaluated at the steady state $\left(x_{1 s s}^{(4)}, \lambda_{1 s s}^{(4)}, x_{2 s s}^{(4)}, \lambda_{2 s s}^{(4)}\right)$ and the fourth-order identity matrix, respectively.

The characterization of the optimal time-paths of the prices and cumulative adoption of both products in the case of the integrated monopolist follows the same steps as previously described for the scenario of two independent producers.

The objective in the case of the integrated monopolist is to choose the prices, $p_{1}$ and $p_{2}$, in order to maximize the following functional:

$$
\int_{0}^{\infty} e^{-r t}\left[\left(p_{1}(t)-c_{1}\right) \dot{x}_{1}(t)+\left(p_{2}(t)-c_{2}\right) \dot{x}_{2}(t)\right] d t
$$

taking into account the differential equations (6) and (7).

The current-value Hamiltonian reads ${ }^{16}$ :

$$
\begin{aligned}
H^{m}\left(x_{1}, p_{1}, x_{2}, p_{2}, \lambda_{1}^{m}, \lambda_{2}^{m}\right) & =\left(p_{1}-c_{1}\right) \dot{x}_{1}+\left(p_{2}-c_{2}\right) \dot{x}_{2}+\lambda_{1}^{m} \dot{x}_{1}+\lambda_{2}^{m} \dot{x}_{2} \\
& =\left(p_{1}-c_{1}+\lambda_{1}^{m}\right) \dot{x}_{1}+\left(p_{2}-c_{2}+\lambda_{2}^{m}\right) \dot{x}_{2},
\end{aligned}
$$

where $\lambda_{1}^{m}$ and $\lambda_{2}^{m}$ denote the costate variables associated with $x_{1}$ and $x_{2}$, respectively.

\footnotetext{
${ }^{16}$ The superscript stands for "monopolistic scenario".
} 
The first-order optimality conditions for an interior solution read:

$$
\begin{aligned}
\frac{\partial H^{m}}{\partial p_{i}} & =0, \quad i=1,2, \\
\dot{\lambda}_{i}^{m} & =r \lambda_{i}^{m}-\frac{\partial H^{m}}{\partial x}, \quad \lim _{t \rightarrow \infty} \lambda_{i}^{m}(t) x_{i}(t) e^{-r t}=0 \quad i=1,2, \\
\dot{x}_{1} & =\left(\alpha_{1}-\beta_{1} p_{1}+b_{1} x_{1}\right)\left(M-x_{1}\right), \quad x_{1}(0)=x_{10}, \\
\dot{x}_{2} & =\left(\alpha_{2}-\beta_{2} p_{2}+b_{2} x_{2}\right)\left(x_{1}-x_{2}\right), \quad x_{2}(0)=x_{20} .
\end{aligned}
$$

Assuming that $x_{1}$ and $x_{2}$ are different from $M$ and $x_{1}$, respectively, from the two first optimality conditions the following expressions from the prices can be derived:

$$
p_{i}=\frac{1}{2 \beta_{i}}\left(b_{i} x_{i}+\alpha_{i}+\beta_{i}\left(c_{i}-\lambda_{i}\right)\right), \quad i=1,2 .
$$

Substituting these expressions in the differential equations describing the time evolution of the state and costate variables, these equations read:

$$
\begin{aligned}
\dot{x}_{1}= & \frac{1}{2}\left(M-x_{1}\right)\left(b_{1} x_{1}+\alpha_{1}-\beta_{1}\left(c_{1}-\lambda_{1}\right)\right), \\
\dot{x}_{2}= & \frac{1}{2}\left(x_{1}-x_{2}\right)\left(b_{2} x_{2}+\alpha_{2}-\beta_{2}\left(c_{2}-\lambda_{2}\right)\right), \\
\dot{\lambda}_{1}= & \frac{1}{4 \beta_{1} \beta_{2}}\left[\beta_{2}\left(b_{1}^{2} x_{1}\left(3 x_{1}-2 M\right)+\left(\alpha_{1}-\beta_{1}\left(c_{1}-\lambda_{1}\right)\right)\left(\alpha_{1}-\beta_{1}\left(c_{1}-\lambda_{1}\right)-2 b_{1}\left(M-2 x_{1}\right)\right)+4 r \beta_{1} \lambda_{1}\right)\right. \\
& \left.-\beta_{1}\left(b_{2} x_{2}+\alpha_{2}-\beta_{2}\left(c_{2}-\lambda_{2}\right)\right)^{2}\right], \\
\dot{\lambda}_{2}= & \frac{1}{4 \beta_{2}}\left[b_{2}^{2} x_{2}\left(3 x_{2}-2 x_{1}\right)+\left(\alpha_{2}-\beta_{2}\left(c_{2}-\lambda_{2}\right)\right)\left(\alpha_{2}-\beta_{2}\left(c_{2}-\lambda_{2}\right)-2 b_{2}\left(x_{1}-2 x_{2}\right)\right)+4 r \beta_{2} \lambda_{2}\right] .
\end{aligned}
$$

The characterization of the steady-states and the analysis of their stability follow the same steps as the analysis developed in the case of the independent producers. Four steady states can be characterized and the numerical simulations focus on the only steady state for which there is a unique optimal path converging to this steady state. This steady-state reads $\left(x_{1 s s}^{(m)}, \lambda_{1 s s}^{(m)}, x_{2 s s}^{(m)}, \lambda_{2 s s}^{(m)}\right)$ with $x_{1 s s}^{(m)}=x_{2 s s}^{(m)}=M$, and

$$
\begin{aligned}
\lambda_{1 s s}^{(m)} & =-\frac{1}{\beta_{1} \beta_{2}}\left(\alpha_{1}-c_{1} \beta_{1}+2 r+b_{1} M-2 \sqrt{r \Gamma}\right), \\
\lambda_{2 s s}^{(m)} & =-\frac{1}{\beta_{2}}\left(\alpha_{2}-c_{2} \beta_{2}+2 r+b_{2} M-2 \sqrt{r\left(\alpha_{2}-c_{2} \beta_{2}+r+b_{2} M\right)}\right),
\end{aligned}
$$

with $\Gamma$ given by:

$$
\begin{aligned}
\Gamma= & \beta_{2}\left[\left(\alpha_{2}-c_{2} \beta_{2}+2 r+b_{2} M-2 \sqrt{r\left(\alpha_{2}-c_{2} \beta_{2}+r+b_{2} M\right)}\right) \beta_{1}\right. \\
& \left.+\left(\alpha_{1}-c_{1} \beta_{1}+r+b_{1} M\right) \beta_{2}\right] .
\end{aligned}
$$


The eigenvalues of the Jacobian matrix evaluated at this steady-state are

$$
\begin{aligned}
& \mu_{1}=r-\sqrt{r\left(\alpha_{2}-c_{2} \beta_{2}+r+b_{2} M\right)}, \\
& \mu_{2}=r-\frac{1}{\beta_{2}} \sqrt{r \Gamma} .
\end{aligned}
$$

The eigenvectors associated are $\bar{v}_{1}^{m}=\left(0, v_{1}^{(m 2)}, v_{1}^{(m 3)}, 1\right)$ and $\bar{v}_{2}^{m}=\left(v_{2}^{(m 1)}, v_{2}^{(m 2)}, v_{2}^{(m 3)}, 1\right)$, with $v_{i}^{(m j)}$ the $j$-th component of vector $\bar{v}_{i}^{m}$ (omitted for brevity). The solution of the system of differential equations read:

$$
\begin{aligned}
& x_{1}(t)=\left(x_{10}-M\right) e^{\mu_{2} t}+M, \\
& \lambda_{1}(t)=\xi v_{1}^{(m 2)} e^{\mu_{1} t}+\psi v_{2}^{(m 2)} e^{\mu_{2} t}+\lambda_{1 s s}^{(m)}, \\
& x_{2}(t)=\xi v_{1}^{(m 3)} e^{\mu_{1} t}+\psi v_{2}^{(m 3)} e^{\mu_{2} t}+M, \\
& \lambda_{2}(t)=\xi e^{\mu_{1} t}+\psi e^{\mu_{2} t}+\lambda_{2 s s}^{(m)},
\end{aligned}
$$

where

$$
\xi=\frac{\left(x_{20}-M\right) v_{2}^{(m 1)}-\left(x_{10}-M\right) v_{2}^{(m 3)}}{v_{1}^{(m 3)} v_{2}^{(m 1)}}, \quad \psi=\frac{x_{10}-M}{v_{1}^{(m 3)}} .
$$

\section{References}

[1] Bass, F. M. (1969). "A New Product Growth for Model Consumer Durables." Management Science, 15(5), 215-227.

[2] Bass, F. M., Krishnan, T. V., \& Jain, D. C. (1994). "Why the Bass Model Fits Without Decision Variables." Marketing Science, 13(3), 203.

[3] Dockner, E., \& Jorgensen, S. (1988). "Optimal Advertising Policies for Diffusion Models of New Product Innovation in Monopolistic Situations." Management Science, 34(1), 119-130.

[4] Horsky, D. (1990). "A Diffusion Model Incorporating Product Benefits, Price, Income and Information." Marketing Science, 9(4), 279-365.

[5] Horsky, Dan, \& Simon, L.S. (1983). "Advertising and the Diffusion of New Products." Marketing Science, 2 (1), 1-17.

[6] Jones, J. M., \& Ritz, C. J. (1991). "Incorporating Distribution into New Product Diffusion Models." International Journal of Research in Marketing, 8(2), 91-112.

[7] Jørgensen, S., \& Zaccour, G. 2004. Differential Games in Marketing. International Series in Quantitative Marketing, Kluwer Academic Publishers. 
[8] Kalish, S. (1985). "A New Product Adoption Model With Price, Advertising, and Uncertainty". Management Science, 31(12), 1569-1585.

[9] Kort, P.M., Taboubi, S. \& Zaccour, G. (2018). "Pricing Decisions in Marketing Channels in the Presence of Optional Contingent Products". Central European Journal of Operations Research. https://doi.org/10.1007/s10100-018-0527-x

[10] Kotler, P.1988. Marketing Management, 6th ed., Prentice Hall, Englewood Cliffs.

[11] Mahajan, V. \& Muller, E. (1991). "Pricing and Diffusion of Primary and Contingent Products." Technological Forecasting and Social Change, 39, 291-307.

[12] Mahajan, V., Muller, E., \& Bass, F. M. (1990). "New Product Diffusion Models in Marketing: A Review and Directions for Research." Journal of Marketing, 54(1), 1-22.

[13] Mahajan, V., Peterson, R. A., Jain, A. K., \& Malhotra, N. (1979). "A New Product Growth Model with a Dynamic Market Potential." Long Range Planning, 12(4), 51-58.

[14] Mesak, H. I. (1996). "Incorporating Price, Advertising and Distribution in Diffusion Models of Innovation: Some Theoretical and Empirical Results." Computers $\&$ Operations Research, 23(10), 1007-1023.

[15] Moorthy, K. S. (1993). "Theoretical Modeling in Marketing." Journal of Marketing, $57(2), 92-106$.

[16] Peres, R., Muller, E., \& Mahajan, V. (2010). "Innovation Diffusion and New Product Growth Models: A Critical Review and Research Directions." International Journal of Research in Marketing, 27(2), 91-106.

[17] Peterson, R.A., \& Mahajan, V. (1978). "Multi-product Growth Models." Research in Marketing, S. Jagdish ed., JAI Press 201-231.

[18] Robinson, B., \& Lakhani, C. (1975). "Dynamic Price Models for New Product Planning." Management Science, 21(10), 1113-1122.

[19] Teng, J., \& Thompson, G. L. (1983). "Oligopoly Models for Optimal Advertising when Production Costs Obey a Learning Curve". Management Science, 29(9), 1087-1101.

[20] Thompson, G. L., \& Teng, J. (1984). "Optimal Pricing and Advertising Policies for New Product Oligopoly Models". Marketing Science, 3(2), 148-168. 


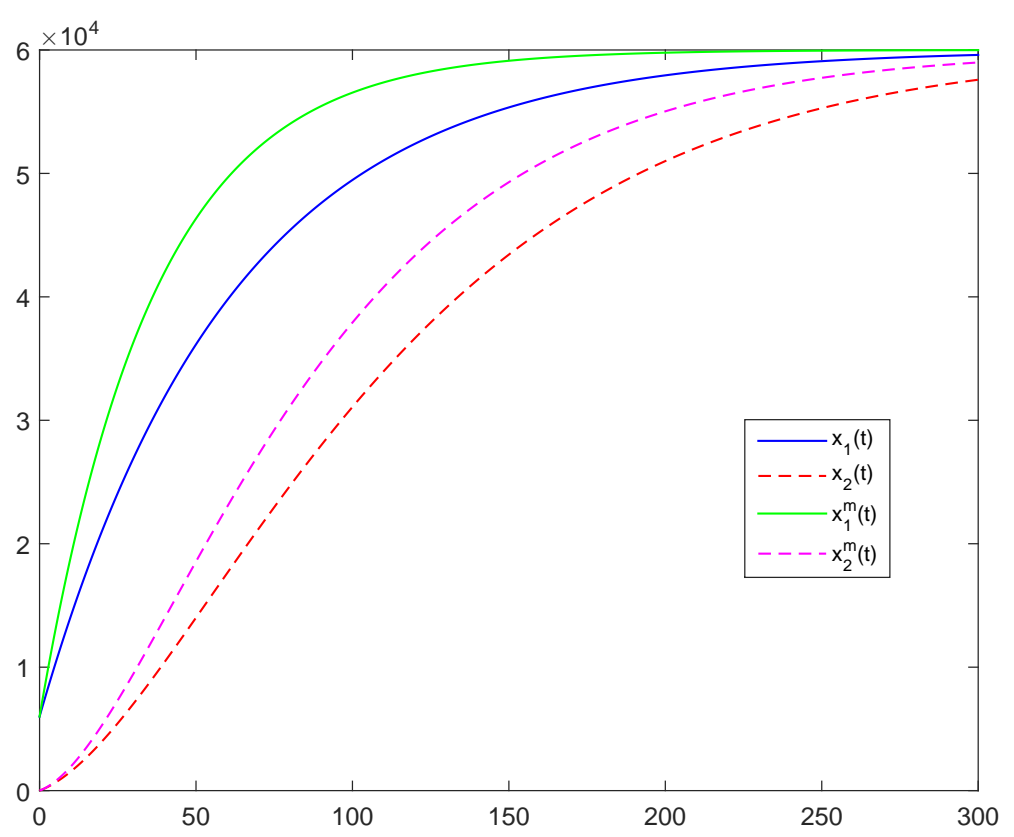

Figure 1: Modified Mahajam \& Muller's model. Comparison cumulative adoption base and contingent products under integrated monopolistic and independent producers. 


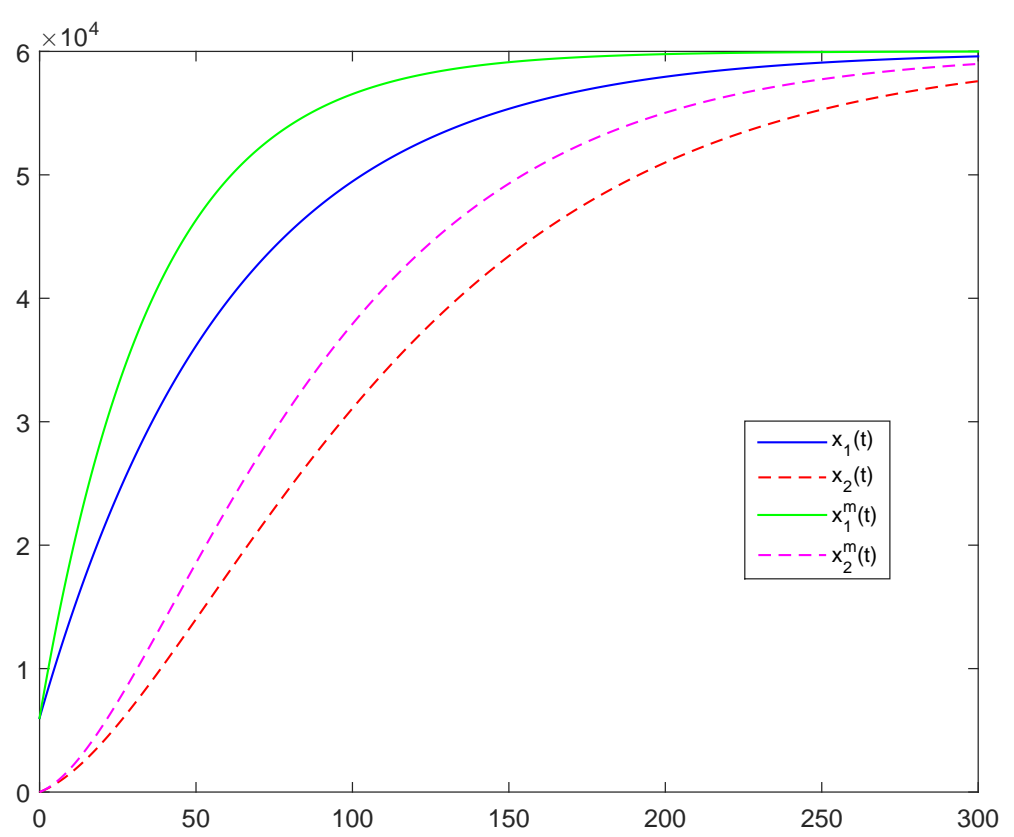

Figure 2: Modified Mahajam \& Muller's model. Comparison retail prices of the base and contingent products under integrated monopolistic and independent producers.

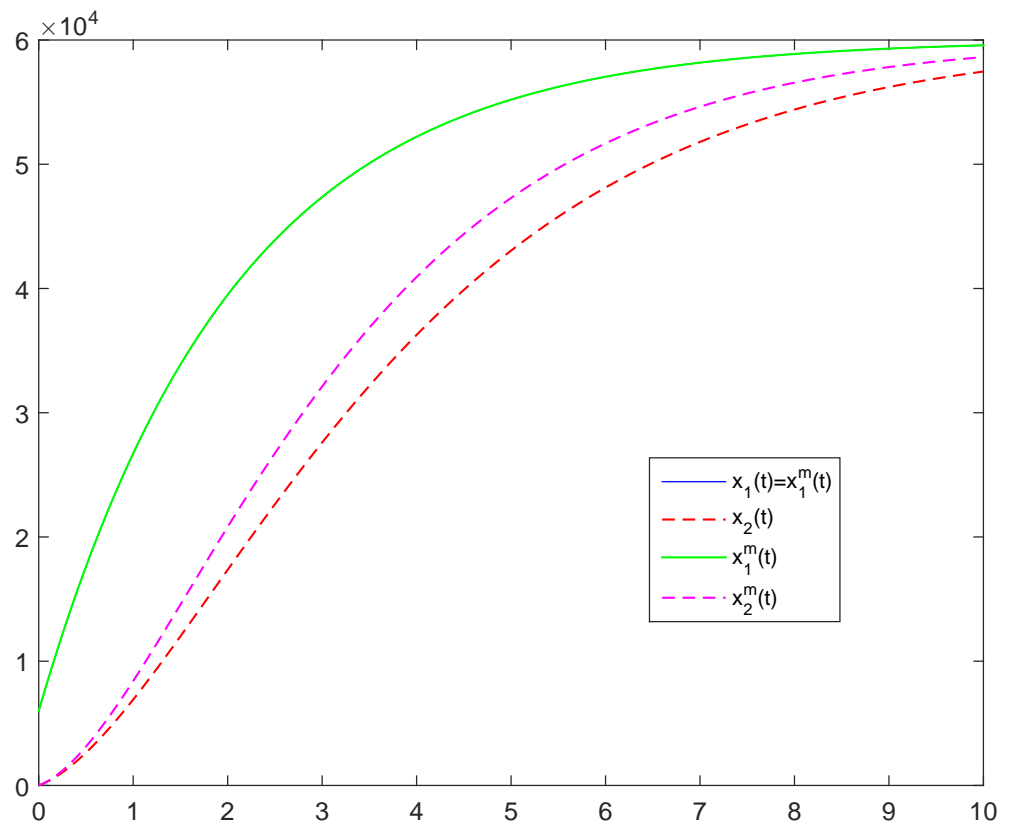

Figure 3: Our model. Comparison cumulative adoption base and contingent products under integrated monopolistic and independent producers. 


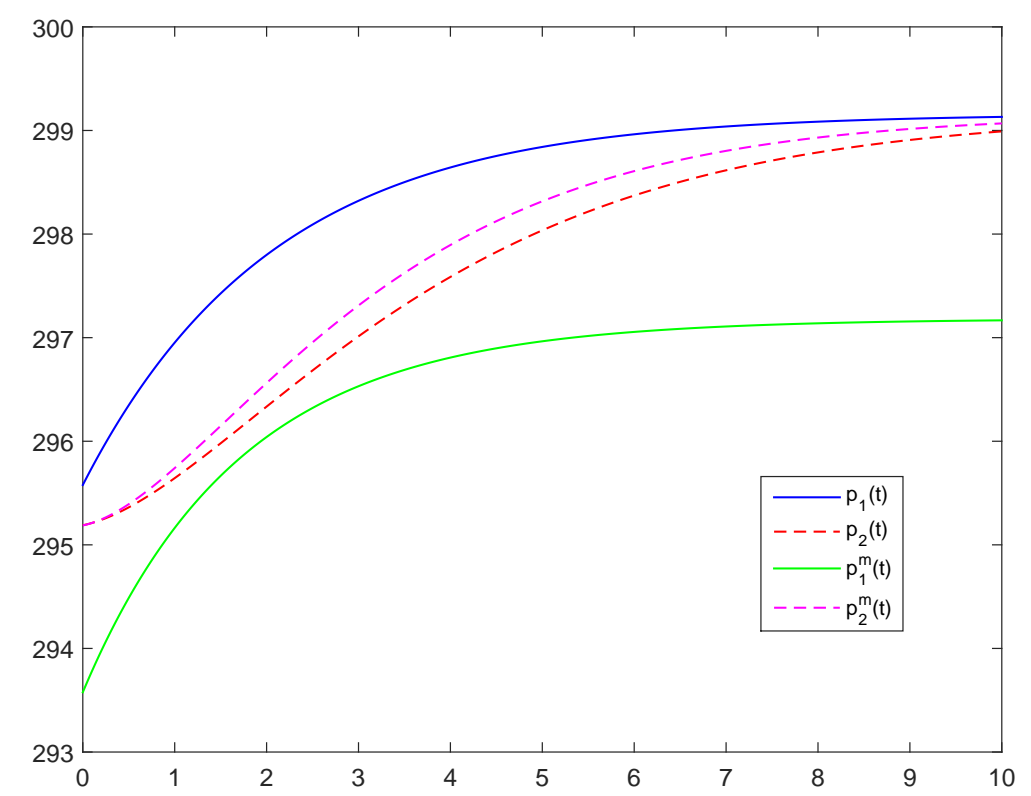

Figure 4: Our model. Comparison retail prices of the base and contingent products under integrated monopolistic and independent producers 